\title{
EXTINCTION OF BRANCHING SYMMETRIC $\alpha$-STABLE PROCESSES
}

\author{
YUICHI SHIOZAWA, * Tohoku University
}

\begin{abstract}
We give a criterion for extinction or local extinction of branching symmetric $\alpha$-stable processes in terms of the principal eigenvalue for time-changed processes of symmetric $\alpha$-stable processes. Here the branching rate and the branching mechanism are spatially dependent. In particular, the branching rate is allowed to be singular with respect to the Lebesgue measure. We apply this criterion to some branching processes.
\end{abstract}

Keywords: Branching process; extinction; local extinction; Brownian motion; symmetric $\alpha$-stable process; time change; principal eigenvalue; gaugeability

2000 Mathematics Subject Classification: Primary 60J80

Secondary 60G52

\section{Introduction}

In this paper, we consider the extinction problem for branching symmetric $\alpha$-stable processes $(0<\alpha \leq 2)$ in which each of the particles moves independently according to the law of the absorbing symmetric $\alpha$-stable process on an open set. The branching rate and the branching mechanism are spatially dependent. In particular, the branching rate is allowed to be singular with respect to the Lebesgue measure. We give a necessary and sufficient condition for extinction in terms of the principal eigenvalue for processes of symmetric $\alpha$-stable processes time changed with respect to the positive continuous additive functional associated with the branching rate and the branching mechanism (Theorem 3.1). We also give a necessary and sufficient condition for local extinction in terms of the principal eigenvalue for time-changed processes (Theorem 3.2).

Sevast'yanov [12, Theorem 4] and Watanabe [19, Theorem 2.2] considered the extinction problem for branching Brownian motions on an open set with spatially independent branching rates and branching mechanisms, and gave a criterion in terms of the principal eigenvalue of the Dirichlet Laplacian. Our approach is different from theirs: they used the ground-state of the Dirichlet Laplacian, while we use the ground-state for the time-changed process (see [17] for a proof of its existence). Moreover, they assumed that the open set is bounded, while we allow the open set to be unbounded. We assume that the branching rate belongs to a certain Kato class, $\mathcal{K}_{\infty}^{D}$ (see Section 2 for the definition). This assumption implies that the branching rate is small at $\infty$. For a bounded open set, the constant branching rate belongs to $\mathcal{K}_{\infty}^{D}$. Hence, our result becomes an extension of theirs even for branching Brownian motions.

Pinsky [11] gave a criterion for the local extinction of measure-valued branching diffusion processes in terms of the generalized principal eigenvalue for Schrödinger operators (see [10, p. 147] for a definition), using the partial differential equation method (see also [5]). On the other

Received 24 January 2006; revision received 9 May 2006.

* Postal address: Mathematical Institute, Tohoku University, Aoba, Sendai, 980-8578, Japan.

Email address: sa0m15@math.tohoku.ac.jp

Dedicated to Professor Masatoshi Fukushima on the occasion of his seventieth birthday. 
hand, Engländer and Kyprianou [4] gave a criterion for the local extinction of branching and measure-valued branching diffusion processes probabilistically. In these papers, the groundstate of the Schrödinger operator plays an essential role. The existence of this ground-state was proved in [10, p. 145, Theorem 3.1] using Harnack's inequality and Schauder's estimate; however, this approach is not applicable to jump processes, because of the nonlocality. To overcome this difficulty, we use the principal eigenvalue and the ground-state for time-changed processes.

We apply the criterion above to some branching symmetric $\alpha$-stable processes. For example, consider the one-dimensional case with $1<\alpha \leq 2$. Take the Dirac measure at $a>0$ as the branching rate. Suppose that each particle splits into two upon branching and dies upon arriving at 0 . We then see, in Example 4.3 below, that this branching symmetric $\alpha$-stable process becomes extinct if and only if

$$
0<a \leq\left(-\frac{\Gamma(\alpha) \cos (\pi \alpha / 2)}{2}\right)^{1 /(\alpha-1)}
$$

We can extend the criterion for extinction or local extinction to branching symmetric Hunt processes if the ground-state exists for time-changed processes of symmetric Hunt processes (Theorem 3.3 and Theorem 3.4). For instance, it is applicable to branching Brownian motions on Riemannian manifolds (Example 4.2). However, it seems hard in general to show the existence of the ground-state. We thus give a sufficient condition for extinction or local extinction without assuming the existence of the ground-state (Theorem 3.5). To do so we use the notion of gaugeability, that is, the exponential integrability of positive continuous additive functionals. Chen [3, Theorem 5.1] and Takeda [15, Theorem 2.4] proved that a measure $\mu \in \mathcal{K}_{\infty}^{D}$ is gaugeable if and only if the principal eigenvalue for the time-changed process with respect to $\mu$ is greater than 1 (see also [18, Theorem 3.1]). By applying this, we establish our sufficient condition. The condition on the gaugeability above says that the principal eigenvalue measures the size of $\mu$. For branching processes, this quantity shows the degree of increase in the number of particles.

\section{Preliminaries}

\subsection{Time changes of symmetric Hunt processes}

Let $X$ be a locally compact, separable metric space and $m$ a positive Radon measure on $X$ with full support. Denote by $\Delta$ the cemetary point. Let $\mathbf{M}=\left(\Omega, \mathcal{F}, \mathcal{F}_{t}, X_{t}, \mathrm{P}_{x}, \zeta\right)$ be an $m$-symmetric transient Hunt process on $X$, where $\left\{\mathcal{F}_{t}\right\}_{t \geq 0}$ is the minimal admissible filtration and $\zeta$ is the lifetime, $\zeta=\inf \left\{t>0: X_{t}=\Delta\right\}$. Suppose that the transition density of $\mathbf{M}$ is absolutely continuous with respect to $m$. Denote by $(\mathcal{E}, \mathcal{F})$ the regular Dirichlet form on $L^{2}(X ; m)$ generated by M. Let $\&$ be the set of smooth measures (see [6, p. 80] for a definition) and denote by $A_{t}^{\mu}$ the positive continuous additive functional in the Revuz correspondence to $\mu \in \delta$ (see [6, Theorem 5.1.4]). Let $\tau_{t}^{\mu}=\inf \left\{s>0: A_{s \wedge \zeta}^{\mu}>t\right\}$. Then the fine support of $\mu$ defined by $F^{\mu}=\left\{x \in X: \mathrm{P}_{x}\left(\tau_{0}^{\mu}=0\right)=1\right\}$ is finely closed and $A_{t}^{\mu}(\omega)$ increases only when $X_{t}(\omega) \in F^{\mu}$ for almost every $\omega \in \Omega$ [6, Lemma 5.1.11]. The process $Y_{t}^{\mu}:=X_{\tau_{t}^{\mu}}$ is called the time-changed process of $X_{t}$ with respect to $A_{t}^{\mu}$, and is a $\mu$-symmetric Hunt process on $F^{\mu}$ with lifetime $A_{\zeta}^{\mu}[6$, Theorem 6.2.1].

We now introduce a class of measures. Let $\delta_{1}$ be the set of positive Radon measures in $\delta$ and $G(x, y)$ the Green's function of $\mathbf{M}$. 
Definition 2.1. A measure $\mu \in \oiint_{1}$ is said to be in $\mathcal{K}_{\infty}$ if, for any $\varepsilon>0$, there exist a compact set $K \subset X$ and a constant $\delta>0$ such that

$$
\sup _{x \in X} \int_{X \backslash K} G(x, y) \mu(\mathrm{d} y)<\varepsilon,
$$

and if, for all measurable sets $B \subset K$ with $\mu(B)<\delta$,

$$
\sup _{x \in X} \int_{B} G(x, y) \mu(\mathrm{d} y)<\varepsilon .
$$

For any $\mu \in \mathcal{K}_{\infty}$, it is known (see [3, Proposition 2.2]) that

$$
\sup _{x \in X} \mathrm{E}_{x}\left[A_{\zeta}^{\mu}\right]=\sup _{x \in X} \int_{X} G(x, y) \mu(\mathrm{d} y)<\infty .
$$

\subsection{Branching symmetric Hunt processes}

Following [8] and [9], we introduce the notion of branching symmetric Hunt processes. Let $\left\{p_{n}(x)\right\}_{n \geq 0}, x \in X$, be a sequence such that $0 \leq p_{n}(x) \leq 1$ and $\sum_{n=0}^{\infty} p_{n}(x)=1$. For $\mu \in \varsigma_{1}$, we denote by $Z$ the random variable of the exponential distribution with rate $A_{t}^{\mu}$ :

$$
\mathrm{P}_{x}\left(t<Z \mid \mathcal{F}_{\infty}\right)=\exp \left(-A_{t}^{\mu}\right) .
$$

A particle of the branching symmetric Hunt process starts at $x \in X$ according to the law $\mathrm{P}_{x}$. When $t=\zeta<Z$, it dies. On the other hand, when $t=Z<\zeta$, it splits into $n$ particles with probability $p_{n}\left(X_{Z_{-}}\right)$. Each of these particles then starts at $X_{Z-}$ independently according to the law $\mathrm{P}_{X_{Z_{-}}}$. Let $X^{(0)}=\{\Delta\}$ and $X^{(1)}=X$. We define the equivalence relation ' $\sim$ ' on $X^{n}$ as follows. Let $\boldsymbol{x}^{n}=\left(x^{1}, x^{2}, x^{3}, \ldots, x^{n}\right), \boldsymbol{y}^{n}=\left(y^{1}, y^{2}, y^{3}, \ldots, y^{n}\right) \in X^{n}$. If there exists a permutation, $\sigma$, of $\{1,2,3, \ldots, n\}$ such that $y^{i}=x^{\sigma(i)}$ for all $i$, then we write $\boldsymbol{x}^{n} \sim \boldsymbol{y}^{n}$. Let $X^{(n)}=X^{n} / \sim$ and $\mathbf{X}=\bigcup_{n=0}^{\infty} X^{(n)}$. When the branching process consists of $n$ particles at time $t$, they define a point in $X^{(n)}$. Hence, it is a branching symmetric Hunt process $\overline{\mathbf{M}}=\left(\boldsymbol{X}_{t}, \mathbf{P}_{\boldsymbol{x}}\right)$ on $\mathbf{X}$ with motion component $\mathbf{M}$, branching rate $\mu$, and branching mechanism $\left\{p_{n}(x)\right\}_{n \geq 0}$. Let $T$ be the first splitting time of $\overline{\mathbf{M}}$ :

$$
\mathbf{P}_{x}(t<T \mid \sigma(X))=\mathrm{P}_{x}\left(t<Z \mid \mathcal{F}_{\infty}\right)=\exp \left(-A_{t}^{\mu}\right) .
$$

Denote by $Z_{t}$ the number of particles of $\overline{\mathbf{M}}$ at time $t$, that is, $Z_{t}=n$ if $\boldsymbol{X}_{t}=\left(\mathrm{X}_{t}^{1}, \mathrm{X}_{t}^{2}, \mathrm{X}_{t}^{3}, \ldots\right.$, $\left.\mathrm{X}_{t}^{n}\right) \in X^{(n)}$. Then $e_{0}:=\inf \left\{t>0: Z_{t}=0\right\}$ is called the extinction time of $\overline{\mathbf{M}}$. Let $u_{\mathrm{e}}(x)=$ $\mathbf{P}_{x}\left(e_{0}<\infty\right)=\mathbf{P}_{x}\left(\lim _{t \rightarrow \infty} Z_{t}=0\right)$. We say that $\overline{\mathbf{M}}$ extincts if $u_{\mathrm{e}} \equiv 1$ on $X$. Denote by $Z_{t}(A)$ the number of particles in $A \subset X$ at time $t$, and let $L_{A}=\sup \left\{t>0: Z_{t}(A)>0\right\}$. We say that $\overline{\mathbf{M}}$ extincts locally if, for any relatively compact open set $A \subset X, \mathbf{P}_{x}\left(L_{A}<\infty\right)=1$ for all $x \in X$.

\subsection{Symmetric $\alpha$-stable processes}

Let $\mathbf{M}^{\alpha}=\left(\Omega, \mathcal{F}, \mathscr{F}_{t}, X_{t}, \mathrm{P}_{x}\right), 0<\alpha \leq 2$, be a symmetric $\alpha$-stable process on $\mathbb{R}^{d}$. Denote by $\left(\mathcal{E}^{\alpha}, \mathcal{F}^{\alpha}\right)$ the Dirichlet form on $L^{2}\left(\mathbb{R}^{d} ; \mathrm{d} x\right)$ generated by $\mathbf{M}^{\alpha}$ :

$$
\begin{aligned}
\mathcal{E}^{\alpha}(f, f) & = \begin{cases}\frac{1}{2} \int_{\mathbb{R}^{d}}|\nabla f|^{2} \mathrm{~d} x, & \alpha=2, \\
\mathcal{A}(d, \alpha) \iint_{\mathbb{R}^{d} \times \mathbb{R}^{d}} \frac{(f(x)-f(y))^{2}}{|x-y|^{d+\alpha}} \mathrm{d} y \mathrm{~d} x, & 0<\alpha<2,\end{cases} \\
\mathcal{F}^{\alpha} & =\left\{f \in L^{2}\left(\mathbb{R}^{d} ; \mathrm{d} x\right): \mathcal{E}^{\alpha}(f, f)<\infty\right\},
\end{aligned}
$$


where

$$
\mathcal{A}(d, \alpha)=\frac{\alpha 2^{\alpha-3} \Gamma((d+\alpha) / 2)}{\pi^{d / 2} \Gamma(1-\alpha / 2)}, \quad \Gamma(x)=\int_{0}^{\infty} \mathrm{e}^{-t} t^{x-1} \mathrm{~d} t .
$$

Let $\mathbf{M}^{D}=\left(X_{t}^{D}, \mathrm{P}_{x}^{D}\right)$ be the absorbing symmetric $\alpha$-stable process on an open set $D \subset \mathbb{R}^{d}$, and denote by $\mathcal{K}_{\infty}^{D}$ the class $\mathcal{K}_{\infty}$ for $\mathbf{M}^{D}$. Let

$$
\lambda(\mu, v ; D)=\inf \left\{\mathcal{E}^{\alpha}(f, f)+\int_{D} f^{2} \mathrm{~d} \mu: f \in C_{0}^{\infty}(D), \int_{D} f^{2} \mathrm{~d} v=1\right\}
$$

for $\mu, v \in \mathcal{K}_{\infty}^{D}$. We then see from [17, Section 4] that $\lambda(\mu, v ; D)$ is the principal eigenvalue for the time-changed process of the $\exp \left(-A_{t}^{\mu}\right)$-subprocess of $\mathbf{M}^{D}$ with respect to $A_{t}^{v}$.

\section{Extinction and local extinction}

Let $\overline{\mathbf{M}^{D}}=\left(\boldsymbol{X}_{t}, \mathbf{P}_{\boldsymbol{x}}\right)$ be a branching symmetric $\alpha$-stable process with motion component $\mathbf{M}^{D}$, branching rate $\mu \in \mathcal{K}_{\infty}^{D}$, and branching mechanism $\left\{p_{n}(x)\right\}_{n \geq 0}$. We first consider the extinction problem of $\overline{\mathbf{M}^{D}}$. Let

$$
F(u)(\cdot)=\sum_{n=0}^{\infty} p_{n}(\cdot) u(\cdot)^{n}
$$

and let $\tau_{D}$ be the exit time of $\mathbf{M}^{\alpha}$ from $D$, that is, $\tau_{D}=\inf \left\{t>0: X_{t} \notin D\right\}$. We then have the following proposition.

Proposition 3.1. The function $u_{\mathrm{e}}$ is the minimal solution to

$$
u(x)=\mathrm{E}_{x}\left[\exp \left(-A_{\tau_{D}}^{\mu}\right) ; \tau_{D}<\infty\right]+\mathrm{E}_{x}\left[\int_{0}^{\tau_{D}} \exp \left(-A_{t}^{\mu}\right) F(u)\left(X_{t}\right) \mathrm{d} A_{t}^{\mu}\right], \quad 0 \leq u \leq 1 .
$$

Proof. The strong Markov property of $\overline{\mathbf{M}^{D}}$ implies that

$$
\begin{aligned}
u_{\mathrm{e}}(x)= & \mathbf{P}_{x}\left(e_{0}=\tau_{D}<T, e_{0}<\infty\right)+\mathbf{P}_{x}\left(e_{0}=T<\tau_{D}, e_{0}<\infty\right) \\
& +\mathbf{P}_{x}\left(T<e_{0} \wedge \tau_{D}, e_{0}<\infty\right) \\
= & \mathbf{P}_{x}\left(\tau_{D}<T, \tau_{D}<\infty\right)+\mathbf{P}_{x}\left(e_{0}=T<\tau_{D}, e_{0}<\infty\right) \\
& +\mathbf{E}_{x}\left[\mathbf{P}_{X_{T}}\left(e_{0}<\infty\right) ; T<e_{0} \wedge \tau_{D}\right] .
\end{aligned}
$$

Since

$$
\begin{aligned}
\mathbf{P}_{x}\left(\tau_{D}<T, \tau_{D}<\infty\right) & =\mathrm{E}_{x}\left[\exp \left(-A_{\tau_{D}}^{\mu}\right) ; \tau_{D}<\infty\right], \\
\mathbf{P}_{x}\left(e_{0}=T<\tau_{D}, e_{0}<\infty\right) & =\mathrm{E}_{x}\left[\int_{0}^{\tau_{D}} \exp \left(-A_{t}^{\mu}\right) p_{0}\left(X_{t}\right) \mathrm{d} A_{t}^{\mu}\right], \\
\mathbf{E}_{x}\left[\mathbf{P}_{X_{T}}\left(e_{0}<\infty\right) ; T<e_{0} \wedge \tau_{D}\right] & =\mathrm{E}_{x}\left[\int_{0}^{\tau_{D}} \exp \left(-A_{t}^{\mu}\right) \sum_{n=1}^{\infty} p_{n}\left(X_{t}\right) u_{\mathrm{e}}\left(X_{t}\right)^{n} \mathrm{~d} A_{t}^{\mu}\right],
\end{aligned}
$$

the function $u_{\mathrm{e}}$ satisfies (3.1).

Let $R=\inf \left\{t>0: Z_{t} \neq Z_{0}\right\}$ and $S=R \wedge T$. Define $S_{0}=0$ and $S_{k}=S_{k-1}+S \circ \theta_{S_{k-1}}$, $k \geq 1$, where $\theta_{t}$ is the shift of paths for $\overline{\mathbf{M}^{D}}$. If $Z_{S_{k}}=0$ for some $k \geq 1$ then we define $S_{l}=S_{k}$ 
for all $l \geq k$. Let $u_{k}(x)=\mathbf{P}_{x}\left(Z_{S_{k}}=0, e_{0}<\infty\right)$. Then $u_{0} \equiv 0$ and

$$
\begin{aligned}
u_{k}(x)= & \mathbf{P}_{x}\left(\tau_{D}<T, \tau_{D}<\infty\right)+\mathbf{P}_{x}\left(e_{0}=T<\tau_{D}, e_{0}<\infty\right) \\
& +\mathbf{E}_{x}\left[\mathbf{P}_{\boldsymbol{X}_{T}}\left(Z_{S_{k-1}}=0, e_{0}<\infty\right) ; T<e_{0} \wedge \tau_{D}\right]
\end{aligned}
$$

Let $\boldsymbol{x}^{n}=\left(x^{1}, x^{2}, x^{3}, \ldots, x^{n}\right) \in D^{(n)}$. Since

$$
\mathbf{P}_{\boldsymbol{x}^{n}}\left(Z_{S_{k-1}}=0, e_{0}<\infty\right) \leq \prod_{i=1}^{n} \mathbf{P}_{x^{i}}\left(Z_{S_{k-1}}=0, e_{0}<\infty\right)
$$

the last term on the right-hand side of (3.2) is not greater than

$\mathbf{E}_{x}\left[\prod_{i=1}^{Z_{T}} \mathbf{P}_{\boldsymbol{X}_{T}^{i}}\left(Z_{S_{k-1}}=0, e_{0}<\infty\right) ; T<e_{0} \wedge \tau_{D}\right]=\mathrm{E}_{x}\left[\int_{0}^{\tau_{D}} \exp \left(-A_{t}^{\mu}\right) F\left(u_{k-1}\right)\left(X_{t}\right) \mathrm{d} A_{t}^{\mu}\right]$,

and, thus,

$$
u_{k}(x) \leq \mathrm{E}_{x}\left[\exp \left(-A_{\tau_{D}}^{\mu}\right) ; \tau_{D}<\infty\right]+\mathrm{E}_{x}\left[\int_{0}^{\tau_{D}} \exp \left(-A_{t}^{\mu}\right) F\left(u_{k-1}\right)\left(X_{t}\right) \mathrm{d} A_{t}^{\mu}\right] .
$$

Suppose that a function $v$ also satisfies (3.1). On account of (3.3), $u_{k} \leq v$ for any $k \geq 1$, by induction, which implies that $\lim _{k \rightarrow \infty} u_{k}=u_{\mathrm{e}} \leq v$.

Lemma 3.1. Any solution to (3.1) is finely continuous.

Proof. Let $u$ be a solution to (3.1). Then the Markov property of $\mathbf{M}^{D}$ yields

$$
\begin{aligned}
u\left(X_{t}\right)= & \exp \left(A_{t}^{\mu}\right) \mathrm{E}_{x}\left[\exp \left(-A_{\tau_{D}}^{\mu}\right) ; \tau_{D}<\infty \mid \mathcal{F}_{t}\right] \\
& +\exp \left(A_{t}^{\mu}\right) \mathrm{E}_{x}\left[\int_{0}^{\tau_{D}} \exp \left(-A_{s}^{\mu}\right) F(u)\left(X_{s}\right) \mathrm{d} A_{s}^{\mu} \mid \mathcal{F}_{t}\right] \\
& -\exp \left(A_{t}^{\mu}\right) \int_{0}^{t} \exp \left(-A_{s}^{\mu}\right) F(u)\left(X_{s}\right) \mathrm{d} A_{s}^{\mu}, \quad \mathrm{P}_{x}^{D} \text {-almost surely, }
\end{aligned}
$$

for all $x \in D$. Since the right-hand side of this expression is right continuous by the right continuity of the filtration $\left\{\mathcal{F}_{t}\right\}_{t \geq 0}$, the function $u$ is finely continuous by [2, Theorem 4.8].

Lemma 3.2. If $\mathrm{P}_{x}\left(\tau_{D}<\infty\right)<1$ for $x \in D$ then $u_{\mathrm{e}}(x)<1$, that is, the process $\overline{\mathbf{M}^{D}}$ does not extinct.

Proof. Since $\mathrm{E}_{x}\left[\exp \left(-A_{\tau_{D}}^{\mu}\right) ; \tau_{D}=\infty\right]>0,(3.1)$ implies that

$$
\begin{aligned}
u_{\mathrm{e}}(x) & \leq \mathrm{E}_{x}\left[\exp \left(-A_{\tau_{D}}^{\mu}\right) ; \tau_{D}<\infty\right]+\mathrm{E}_{x}\left[\int_{0}^{\tau_{D}} \exp \left(-A_{t}^{\mu}\right) F(1)\left(X_{t}\right) \mathrm{d} A_{t}^{\mu}\right] \\
& =1-E_{x}\left[\exp \left(-A_{\tau_{D}}^{\mu}\right) ; \tau_{D}=\infty\right]<1 .
\end{aligned}
$$

Let $Q(x)=F^{\prime}(1)(x)=\sum_{n=1}^{\infty} n p_{n}(x)$ and suppose that $\sup _{x \in D} Q(x)<\infty$. Let

$$
\lambda(\mu, Q ; D)=\inf \left\{\mathcal{E}^{\alpha}(f, f)+\int_{D} f^{2} \mathrm{~d} \mu: f \in C_{0}^{\infty}(D), \int_{D} f^{2} Q \mathrm{~d} \mu=1\right\} .
$$


Theorem 3.1. If $\mathrm{P}_{x}\left(\tau_{D}<\infty\right)=1$ for all $x \in D$ then $\overline{\mathbf{M}^{D}}$ extincts if and only if

$$
\lambda(\mu, Q ; D) \geq 1 \text {. }
$$

Proof. Let $\lambda \equiv \lambda(\mu, Q ; D)$. First suppose that $\lambda \geq 1$. Let $u$ be a solution to (3.1). We denote by $\sigma_{A}$ the hitting time of the set $A$ in $D$, that is, $\sigma_{A}=\inf \left\{t>0: X_{t}^{D} \in A\right\}$. Let $O=\{x \in D: u(x)<1\}$ and assume that $\mathrm{P}_{x}^{D}\left(\sigma_{F^{\mu} \cap O}<\infty\right)>0$ for all $x \in D$. Since $u$ is finely continuous, by Lemma 3.1, and $u-u^{n}<(n-1)(1-u)$ on $O$ for $n \geq 2$, it follows from (3.1) and the assumption on $D$ that

$$
\begin{aligned}
u(x)= & \mathrm{E}_{x}\left[\exp \left(-A_{\tau_{D}}^{\mu}\right)\right]+\mathrm{E}_{x}\left[\int_{0}^{\tau_{D}} \exp \left(-A_{t}^{\mu}\right) u\left(X_{t}\right) \mathrm{d} A_{t}^{\mu}\right] \\
& +\mathrm{E}_{x}\left[\int_{0}^{\tau_{D}} \exp \left(-A_{t}^{\mu}\right) \sum_{n=0}^{\infty} p_{n}\left(X_{t}\right)\left(u\left(X_{t}\right)^{n}-u\left(X_{t}\right)\right) \mathrm{d} A_{t}^{\mu}\right] \\
> & \mathrm{E}_{x}\left[\exp \left(-A_{\tau_{D}}^{\mu}\right)\right]+\mathrm{E}_{x}\left[\int_{0}^{\tau_{D}} \exp \left(-A_{t}^{\mu}\right) u\left(X_{t}\right) \mathrm{d} A_{t}^{\mu}\right] \\
& -\mathrm{E}_{x}\left[\int_{0}^{\tau_{D}} \exp \left(-A_{t}^{\mu}\right) \sum_{n=0}^{\infty}(n-1) p_{n}\left(X_{t}\right)\left(1-u\left(X_{t}\right)\right) \mathrm{d} A_{t}^{\mu}\right]
\end{aligned}
$$

for all $x \in D$. Let $v=1-u$. Then the inequality above is equivalent to

$$
0 \leq v(x)<G_{\mu}^{Q \mu} v(x),
$$

where $G_{\mu}^{Q \mu}$ is the generalized resolvent defined by

$$
G_{\mu}^{Q \mu} f(x)=\mathrm{E}_{x}\left[\int_{0}^{\tau_{D}} \exp \left(-A_{t}^{\mu}\right) f\left(X_{t}\right) \mathrm{d} A_{t}^{Q \mu}\right]
$$

for any measurable function $f$ in $D$ such that the right-hand side of the expression makes sense. Let $h$ be the ground-state of $\lambda$, that is, the function attaining the infimum of the right-hand side of (3.4). Then $h$ is bounded, strictly positive, and continuous on $D$ (see [17]). Moreover, for a compact set $K \subset D$ and a fixed point $o \in K$,

$$
h(x) \leq C G^{D}(o, x), \quad x \in D \backslash K,
$$

where $G^{D}(x, y)$ is the Green's function of $\mathbf{M}^{D}$ and $C$ is some constant depending on $K$. Therefore,

$$
\int_{D \backslash K} h(y) \mu(\mathrm{d} y) \leq C \sup _{x \in D} \int_{D \backslash K} G^{D}(x, y) \mu(\mathrm{d} y)<\infty
$$

by the definition of $\mathcal{K}_{\infty}^{D}$, which yields $\int_{D} h(x) \mu(\mathrm{d} x)<\infty$. Since

$$
h(x)=\lambda G_{\mu}^{Q \mu} h(x),
$$

(3.5) shows that

$$
\begin{aligned}
\int_{D} h(x) v(x) Q(x) \mu(\mathrm{d} x) & =\lambda \int_{D} G_{\mu}^{Q \mu} h(x) v(x) Q(x) \mu(\mathrm{d} x) \\
& =\lambda \int_{D} h(x) G_{\mu}^{Q \mu} v(x) Q(x) \mu(\mathrm{d} x) \\
& >\lambda \int_{D} h(x) v(x) Q(x) \mu(\mathrm{d} x),
\end{aligned}
$$


where the second equality follows from the $Q \mu$-symmetry of $G_{\mu}^{Q \mu}$ (see [1, Theorem 3.2(iv)]). This contradicts $\lambda \geq 1$. Hence, $\mathrm{P}_{x}^{D}\left(\sigma_{F^{\mu} \cap O}<\infty\right)=0$ for some $x \in D$, which implies that $\mathrm{P}_{x}^{D}\left(\sigma_{F^{\mu} \cap O}<\infty\right)=0$ for all $x \in D$, by the irreducibility of $\mathbf{M}^{D}$. Accordingly, (3.1) yields $u \equiv 1$, and, thus, $u_{\mathrm{e}} \equiv 1$ by Proposition 3.1 .

Next suppose that $\lambda<1$. Choose a $\beta$ such that $\lambda<\beta<1$ and an $\varepsilon$ such that $0<\varepsilon<1$ and $F^{\prime}(1-\varepsilon) \geq \beta F^{\prime}(1)=\beta Q(x)$. Let $\delta$ be a positive constant such that $\delta \sup _{x \in D} h(x) \leq \varepsilon$, and let $w(x)=1-\delta h(x)$. Then

$$
\begin{aligned}
& \mathrm{E}_{x}\left[\exp \left(-A_{\tau_{D}}^{\mu}\right)\right]+\mathrm{E}_{x}\left[\int_{0}^{\tau_{D}} \exp \left(-A_{t}^{\mu}\right) F(w)\left(X_{t}\right) \mathrm{d} A_{t}^{\mu}\right] \\
& \quad=1-\mathrm{E}_{x}\left[\int_{0}^{\tau_{D}} \exp \left(-A_{t}^{\mu}\right)(F(1)-F(w))\left(X_{t}\right) \mathrm{d} A_{t}^{\mu}\right] \\
& =1-\mathrm{E}_{x}\left[\int_{0}^{\tau_{D}} \exp \left(-A_{t}^{\mu}\right) F^{\prime}(\gamma)\left(X_{t}\right)\left(1-w\left(X_{t}\right)\right) \mathrm{d} A_{t}^{\mu}\right],
\end{aligned}
$$

where $\gamma$ is a function satisfying $1-\varepsilon<w(x)<\gamma(x)<1$ for all $x \in D$. Since $F^{\prime}(\gamma)(x) \geq$ $F^{\prime}(1-\varepsilon)(x) \geq \beta Q(x)$, the right-hand side of (3.7) is not greater than

$$
1-\beta \delta G_{\mu}^{Q \mu} h(x)<1-\delta h(x)=w(x),
$$

by (3.6) and the relation $\beta>\lambda$. Thus,

$$
\mathrm{E}_{x}\left[\exp \left(-A_{\tau_{D}}^{\mu}\right)\right]+\mathrm{E}_{x}\left[\int_{0}^{\tau_{D}} \exp \left(-A_{t}^{\mu}\right) F(w)\left(X_{t}\right) \mathrm{d} A_{t}^{\mu}\right]<w(x) .
$$

On account of (3.3) and (3.8), $u_{k}<w$ for any $k \geq 1$, by induction. Hence, $\lim _{k \rightarrow \infty} u_{k}=u_{\mathrm{e}} \leq$ $w<1$ on $D$.

Remark 3.1. Let $D \subset \mathbb{R}^{d}$ be an open set, let $\mu \in \mathcal{K}_{\infty}^{D}$, and let $\lambda(\mu ; D) \equiv \lambda(0, \mu ; D)$. Suppose that $Q(x) \equiv Q$. Then

$$
\lambda(\mu, Q ; D) \geq 1 \quad \Longleftrightarrow \quad \lambda(\mu ; D) \geq Q-1 .
$$

This says that if $Q \leq 1$ then $\overline{\mathbf{M}^{D}}$ extincts for any branching rate $\mu$ in $\mathcal{K}_{\infty}^{D}$.

Let us denote by Cap the 0-capacity associated with the Dirichlet form of $\mathbf{M}^{\alpha}$.

Lemma 3.3. ([16, Lemma 4.5].) For any closed set $K \subset \mathbb{R}^{d}$ with $\operatorname{Cap}(K)>0$ and any $\mu, v \in \mathcal{K}_{\infty}^{\mathbb{R}^{d} \backslash K}$, we have $\lambda\left(\mu, v ; \mathbb{R}^{d} \backslash K\right)>\lambda\left(\mu, v ; \mathbb{R}^{d}\right)$.

Combining Theorem 3.1 with Lemma 3.3 yields the following corollary.

Corollary 3.1. If $\lambda\left(\mu, Q ; \mathbb{R}^{d}\right) \geq 1$ then $\overline{\mathbf{M}^{D}}$ extincts for each open set $D \subset \mathbb{R}^{d}$ such that $\mathrm{P}_{x}\left(\tau_{D}<\infty\right)=1$ for all $x \in D$.

Let $N_{K}$ be the number of branches of $\overline{\mathbf{M}^{\alpha}}$ ever hitting a closed set $K \subset \mathbb{R}^{d}$. Takeda [16, Theorem 1.2] showed that then, for any closed set $K \subset \mathbb{R}^{d}$ with $\operatorname{Cap}(K)>0$,

$$
\sup _{x \in \mathbb{R}^{d} \backslash K} \mathbf{E}_{x}\left[N_{K}\right]<\infty \quad \Longleftrightarrow \lambda\left(\mu, Q ; \mathbb{R}^{d} \backslash K\right)>1 .
$$


Let $D \subset \mathbb{R}^{d}$ be an open set such that $\mathrm{P}_{x}\left(\tau_{D}<\infty\right)=1$ for all $x \in D$. Then

$$
\begin{array}{llll}
\lambda(\mu, Q ; D)>1 & \Longrightarrow & \mathbf{P}_{x}\left(e_{0}<\infty\right) \equiv 1, & \sup _{x \in D} \mathbf{E}_{x}\left[N_{\mathbb{R}^{d} \backslash D}\right]<\infty, \\
\lambda(\mu, Q ; D)=1 & \Longrightarrow & \mathbf{P}_{x}\left(e_{0}<\infty\right) \equiv 1, & \sup _{x \in D} \mathbf{E}_{x}\left[N_{\mathbb{R}^{d} \backslash D}\right]=\infty, \\
\lambda(\mu, Q ; D)<1 & \Longrightarrow & \mathbf{P}_{x}\left(e_{0}<\infty\right)<1, & \sup _{x \in D} \mathbf{E}_{x}\left[N_{\mathbb{R}^{d} \backslash D}\right]=\infty .
\end{array}
$$

The next lemma says that, for $\mathbf{P}_{x}$-almost every path $\omega$ of $\overline{\mathbf{M}^{D}}, e_{0}(\omega)=\infty$ if and only if $\lim _{t \rightarrow \infty} Z_{t}(\omega)=\infty$.

Lemma 3.4. If $\mathrm{P}_{x}\left(\tau_{D}<\infty\right)=1$ for all $x \in D$ then

$$
\mathbf{P}_{x}\left(\lim _{t \rightarrow \infty} Z_{t}=0 \text { or } \lim _{t \rightarrow \infty} Z_{t}=\infty\right)=1 \text { for all } x \in D .
$$

Proof. We first show that

$$
\mathbf{P}_{\boldsymbol{x}^{k}}\left(Z_{t}=k \text { for all } t \geq 0\right)=0 \quad \text { for all } \boldsymbol{x}^{k} \in D^{(k)},
$$

for any $k \geq 1$. Note that it suffices to consider the case $k=1$. Define $T_{1}=T$ and $T_{n}=$ $T_{n-1}+T \circ \theta_{T_{n-1}}, n \geq 1$. Then $T_{n}$ denotes the $n$th branching time of $\overline{\mathbf{M}^{D}}$. Let $B$ be the total number of particle splits, that is, $B=\sup \left\{n \geq 1: T_{n}<\infty\right\}$, and let $s_{n}(x)=\mathbf{P}_{x}\left(Z_{t}=1\right.$ for all $t \geq 0, B=n)$. Then $s_{0}(x)=0$ by the assumption on $D$, and

$$
s_{n}(x)=\mathrm{E}_{x}\left[\int_{0}^{\tau_{D}} \exp \left(-A_{t}^{\mu}\right) p_{1}\left(X_{t}\right) s_{n-1}\left(X_{t}\right) \mathrm{d} A_{t}^{\mu}\right]=0
$$

by induction. Consequently

$$
\mathbf{P}_{x}\left(Z_{t}=1 \text { for all } t \geq 0, B<\infty\right)=\sum_{n=0}^{\infty} s_{n}(x)=0
$$

for all $x \in D$. Let $t(x)=\mathbf{P}_{x}\left(Z_{t}=1\right.$ for all $\left.t \geq 0, B=\infty\right)$. Then

$$
\begin{aligned}
t(x) & =\mathrm{E}_{x}\left[\int_{0}^{\tau_{D}} \exp \left(-A_{t}^{\mu}\right) p_{1}\left(X_{t}\right) t\left(X_{t}\right) \mathrm{d} A_{t}^{\mu}\right] \\
& =\mathrm{E}_{x}\left[\int_{0}^{\tau_{D}} \exp \left(-A_{t}^{\mu}\right) \frac{\left(A_{t}^{p_{1} \mu}\right)^{n}}{n !} t\left(X_{t}\right) \mathrm{d} A_{t}^{p_{1} \mu}\right]
\end{aligned}
$$

for any $n \geq 1$. Since $\sum_{n=0}^{\infty} t(x)<\infty$, by (2.1), we have $t \equiv 0$ on $D$, whence (3.9) holds.

We next show that the probability that $Z_{t}$ equals $k$ infinitely often (that is, for infinitely many values of $t$ ) is 0 for each $k \geq 1$. For a positive integer $k$, let

$$
\begin{aligned}
U \equiv U_{1} & =\inf \left\{t>0: Z_{t}=k\right\}, & & V_{1}=U_{1}+R \circ \theta_{U_{1}}, \\
U_{n} & =V_{n-1}+U \circ \theta_{V_{n-1}}, & & V_{n}=U_{n}+R \circ \theta_{U_{n}},
\end{aligned}
$$

with inf $\varnothing=\infty$, where $R=\inf \left\{t>0: Z_{t} \neq Z_{0}\right\}$. Then

$$
\mathbf{P}_{x}\left(Z_{t}=k \text { infinitely often }\right)=\lim _{n \rightarrow \infty} \mathbf{P}_{x}\left(U_{n}<\infty\right)
$$


By the strong Markov property of $\overline{\mathbf{M}^{D}}$ and the definition of $V_{1}$,

$$
\begin{aligned}
\mathbf{P}_{x}\left(U_{2}<\infty\right) & =\mathbf{E}_{x}\left[\mathbf{P}_{X_{V_{1}}}(U<\infty) ; V_{1}<\infty\right] \\
& =\mathbf{E}_{x}\left[\mathbf{P}_{X_{U_{1}}}\left(R+U \circ \theta_{R}<\infty\right) ; U_{1}<\infty\right] .
\end{aligned}
$$

Let $\gamma=\exp \left(-\sup _{x \in D} \mathrm{E}_{x}\left[A_{\tau_{D}}^{\mu}\right]\right)$. Then

$$
\mathbf{P}_{x}\left(\tau_{D}<T\right)=\mathrm{E}_{x}\left[\exp \left(-A_{\tau_{D}}^{\mu}\right)\right] \geq \gamma,
$$

by the assumption on $D$ and Jensen's inequality. As a direct calculation yields

$$
\begin{aligned}
\mathbf{P}_{x^{k}}\left(R+U \circ \theta_{R}<\infty\right) & =1-\mathbf{P}_{x^{k}}\left(R+U \circ \theta_{R}=\infty\right) \\
& \leq 1-\prod_{i=1}^{k} \mathbf{P}_{x^{i}}\left(\tau_{D}<T\right) \\
& \leq 1-\gamma^{k}
\end{aligned}
$$

for any $k \geq 1$ and $\boldsymbol{x}^{k} \in D^{(k)}$, it holds that $\mathbf{P}_{x}\left(U_{2}<\infty\right) \leq 1-\gamma^{k}$; thus, $\mathbf{P}_{x}\left(U_{n}<\infty\right) \leq$ $\left(1-\gamma^{k}\right)^{n-1}$ by induction. Noting that $\gamma>0$, by (2.1), we obtain $\lim _{n \rightarrow \infty} \mathbf{P}_{x}\left(U_{n}<\infty\right)=0$, thereby completing the proof.

We next consider the local extinction of $\overline{\mathbf{M}^{D}}$. Let $A$ be a relatively compact open set in $D$ and denote by $\rho_{A}$ the last exit time of $\mathbf{M}^{D}$ from $A$, that is, $\rho_{A}=\sup \left\{t>0: X_{t}^{D} \in A\right\}$. Let $u_{\mathrm{e}}^{A}(x)=\mathbf{P}_{x}\left(L_{A}<\infty\right)$. By arguments similar to those yielding Proposition 3.1, we have the following result.

Proposition 3.2. For each relatively compact open set $A \subset D$, the function $u_{\mathrm{e}}^{A}$ is the solution to

$$
u(x)=\mathrm{E}_{x}\left[\exp \left(-A_{\tau_{D}}^{\mu}\right) ; \rho_{A}<\infty\right]+\mathrm{E}_{x}\left[\int_{0}^{\tau_{D}} \exp \left(-A_{t}^{\mu}\right) F(u)\left(X_{t}\right) \mathrm{d} A_{t}^{\mu}\right], \quad 0 \leq u \leq 1 .
$$

Theorem 3.2. Suppose that, for any relatively compact open set $A \subset D, \mathrm{P}_{x}^{D}\left(\rho_{A}<\infty\right)=1$ for all $x \in D$. Then $\overline{\mathbf{M}^{D}}$ extincts locally if and only if $\lambda(\mu, Q ; D) \geq 1$.

Proof. First suppose that $\lambda(\mu, Q ; D) \geq 1$. We then see, in a similar way to Theorem 3.1, that the solution to (3.10) is uniquely determined by $u \equiv 1$ on $D$, which implies that $u_{\mathrm{e}}^{A} \equiv 1$ on $D$ for each relatively compact open set $A \subset D$. Hence, $\overline{\mathbf{M}^{D}}$ extincts locally. Next suppose that $\lambda(\mu, Q ; D)<1$. Let $G$ be a relatively compact open set in $D$ such that $\lambda(\mu, Q ; G)<1$. Then

$$
\overline{\mathbf{M}^{G}}=\left(\mathbf{P}_{X}^{G}\right)
$$

does not extinct, by Lemma 3.2 or Theorem 3.1. In other words, $\mathbf{P}_{x}^{G}\left(e_{0}=\infty\right)>0$ for some $x \in G$. Since $\mathbf{P}_{x}^{G}\left(e_{0}=\infty\right) \leq \mathbf{P}_{x}\left(L_{G}=\infty\right)$ for $x \in G$, the branching process $\overline{\mathbf{M}^{D}}$ does not extinct locally.

Remark 3.2. Extinction of the branching process implies local extinction. Moreover, if $\mathrm{P}_{x}\left(\tau_{D}<\infty\right)=1$ for all $x \in D$ then extinction and local extinction are equivalent. For example, this holds for any bounded open sets and for $D=\mathbb{R} \backslash\{0\}$ when $d=1$ and $1<\alpha \leq 2$ (see also Example 4.3, below). On the other hand, if we take $D=\mathbb{R}^{d}$ with $1<\alpha \leq 2$ and $d>\alpha$, then the branching process does not extinct, but may extinct locally (see Example 4.4). 
Let $\mathbf{M}=\left(X_{t}, \mathrm{P}_{x}\right)$ be an $m$-symmetric irreducible and transient Hunt process on $X$, where $X$ is a locally compact separable metric space and $m$ is a positive Radon measure on $X$ with full support. We now give a criterion for the extinction or local extinction of general branching symmetric Hunt processes. Let us denote by $\overline{\mathbf{M}}$ the branching symmetric Hunt process with branching rate $\mu \in \mathcal{K}_{\infty}$. Let $Q(x)=\sum_{n=0}^{\infty} n p_{n}(x)$ and suppose that $\sup _{x \in X} Q(x)<\infty$. We now define

$$
\lambda(\mu, Q)=\inf \left\{\mathcal{E}(f, f)+\int_{X} \tilde{f}^{2} d \mu: f \in \mathcal{F}, \int_{X} \tilde{f}^{2} Q \mathrm{~d} \mu=1\right\},
$$

where $\tilde{f}$ is a quasicontinuous modification of $f$ (see [6, p. 68] for a definition).

Assumption 3.1. (Existence of the ground-state.) The infimum of (3.11) is attained by a bounded, strictly positive and continuous function in $L^{1}(X ; \mu)$.

Theorem 3.3. Suppose that $\mathrm{P}_{x}(\zeta<\infty)=1$ for all $x \in X$. Then, under Assumption 3.1, $\overline{\mathbf{M}}$ extincts if and only if $\lambda(\mu, Q) \geq 1$.

Denote by $\rho_{A}$ the last exit time of $\mathbf{M}$ from a set $A$ in $D$, that is, $\rho_{A}=\sup \left\{t>0: X_{t} \in A\right\}$.

Theorem 3.4. Suppose that, for any relatively compact open set $A \subset X, \mathrm{P}_{x}\left(\rho_{A}<\infty\right)=1$ for all $x \in X$. Then, under Assumption 3.1, $\overline{\mathbf{M}}$ extincts locally if and only if $\lambda(\mu, Q) \geq 1$.

Theorem 3.3 and Theorem 3.4 are proved using the same arguments as Theorem 3.1 and Theorem 3.2, respectively. However, it seems hard in general to check Assumption 3.1 for symmetric Hunt processes. Here we give a sufficient condition for extinction or local extinction of $\overline{\mathbf{M}}$ that does not require Assumption 3.1.

Theorem 3.5. Suppose that $\lambda(\mu, Q)>1$. If $\mathrm{P}_{x}(\zeta<\infty)=1$ for all $x \in X$ then $\overline{\mathbf{M}}$ extincts. If, for any relatively compact open set $A \subset X, \mathrm{P}_{x}\left(\rho_{A}<\infty\right)=1$ for all $x \in X$, then $\overline{\mathbf{M}}$ extincts locally.

Proof. Suppose that $\lambda(\mu, Q)>1$ and that $\mathrm{P}_{x}(\zeta<\infty)=1$ for all $x \in X$. Note that Proposition 3.1 holds for $\overline{\mathbf{M}}$ upon replacement of the exit time $\tau_{D}$ in (3.1) by the lifetime $\zeta$. Let $u$ be a solution to this modified version of (3.1), and let $v=1-u$. We can then show that

$$
0 \leq v(x)<\mathrm{E}_{x}\left[\int_{0}^{\zeta} \exp \left(-A_{t}^{\mu}\right) v\left(X_{t}\right) \mathrm{d} A_{t}^{Q \mu}\right]
$$

in a way similar to that yielding (3.5). Since

$$
0 \leq v(x)<\mathrm{E}_{x}\left[\int_{0}^{\zeta} \exp \left(-A_{t}^{\mu}\right) \frac{\left(A_{t}^{Q \mu}\right)^{n}}{n !} \mathrm{d} A_{t}^{Q \mu}\right], \quad n \geq 1,
$$

by the iterations of the inequality (3.12) it holds that

$$
\sum_{n=0}^{\infty} v(x) \leq \mathrm{E}_{x}\left[\int_{0}^{\zeta} \exp \left(A_{t}^{(Q-1) \mu}\right) \mathrm{d} A_{t}^{Q \mu}\right]
$$

Noting that $\lambda(\mu, Q)>1$ if and only if the right-hand side of this expression is uniformly finite [3, Corollary 2.9, Theorem 5.2], we obtain $v \equiv 0$ and $u \equiv 1$ on $X$, which implies that $u_{\mathrm{e}} \equiv 1$ on $X$. In the same way, we can show the local extinction of $\overline{\mathbf{M}}$. 


\section{Examples}

\subsection{Branching Brownian motions}

Example 4.1. Suppose that $d=1$. Let $\mathbf{M}^{v}, v \in \varsigma_{1}$, be the killed Brownian motion with respect to $\exp \left(-A_{t}^{v}\right)$, and let $\overline{\mathbf{M}^{v}}$ be the branching Brownian motion with motion component $\mathbf{M}^{\nu}$ and branching rate $\mu \in \mathcal{K}_{\infty}$. First take $\mu=\delta_{0}$ and $v=\delta_{-a}+\delta_{a}$ for $a>0$. Since

$$
\inf \left\{\frac{1}{2} \int_{-\infty}^{\infty}\left(\frac{\mathrm{d} f}{\mathrm{~d} x}\right)^{2} \mathrm{~d} x+f(-a)^{2}+f(a)^{2}: f \in C_{0}^{\infty}(\mathbb{R}), f(0)^{2}=1\right\}=\frac{2}{1+2 a},
$$

by [13, Example 3.2], the branching process $\overline{\mathbf{M}^{v}}$ extincts if and only if

$$
Q(0) \leq 1+\frac{2}{1+2 a} .
$$

Next take $\mu=\delta_{-b}+\delta_{b}, b>0$, and $v=\delta_{0}$. Suppose that $Q(x) \equiv Q$. Since

$$
\inf \left\{\frac{1}{2} \int_{-\infty}^{\infty}\left(\frac{\mathrm{d} f}{\mathrm{~d} x}\right)^{2} \mathrm{~d} x+f(0)^{2}: f \in C_{0}^{\infty}(\mathbb{R}), f(-b)^{2}+f(b)^{2}=1\right\}=\frac{1}{2(1+b)},
$$

by [13, Example 3.2], the branching process $\overline{\mathbf{M}^{v}}$ extincts if and only if

$$
Q \leq 1+\frac{1}{2(1+b)}
$$

Example 4.2. Let $M$ be a spherically symmetric Riemannian manifold with a pole $o$ and consider the Brownian motion on $M$. Denote by $(\mathcal{E}, \mathcal{F})$ the associated Dirichlet form on $L^{2}(M ; V)$, where $V$ is the Riemannian volume on $M$ :

$$
\begin{gathered}
\qquad \mathcal{E}(f, f)=\frac{1}{2} \int_{M}|\nabla f|^{2} \mathrm{~d} V, \\
\mathcal{F}=\text { the closure of } C_{0}^{\infty}(M) \text { with respect to } \mathcal{E}(\cdot, \cdot)+\|\cdot\|_{L^{2}(M ; V)}^{2} .
\end{gathered}
$$

Let $B(r)=\{x \in M: d(x, o)<r\}$, where $d(\cdot, \cdot)$ is the Riemannian distance on $M$, and $\partial B(r)=\{x \in M: d(x, o)=r\}$. Denote by $\delta_{r}$ the surface measure of $\partial B(r)$ and let

$$
S(r)=\delta_{r}(\partial B(r)) \text { and } G(r)=\int_{r}^{\infty} \frac{1}{S(\rho)} \mathrm{d} \rho .
$$

We now let

$$
\lambda\left(\delta_{R} ; M \backslash B(r)\right)=\inf \left\{\mathcal{E}(f, f): f \in C_{0}^{\infty}(M \backslash B(r)), \int_{\partial B(R)} f^{2} \mathrm{~d} \delta_{R}=1\right\}
$$

for $r$ and $R$ with $R>r>0$. The following results were shown by Takeda (private communication (2005)): if $(Q-1) S(R) G(R)>\frac{1}{2}$ then

$$
\lambda\left(\delta_{R} ; M \backslash B(r)\right) \geq Q-1 \quad \Longleftrightarrow \quad r_{0} \leq r<R,
$$

where the positive constant $r_{0}$ is a unique root of

$$
G(r)=\frac{2(Q-1) S(R) G(R)^{2}}{2(Q-1) S(R) G(R)-1} .
$$

If $(Q-1) S(R) G(R) \leq \frac{1}{2}$ then $\lambda\left(\delta_{R} ; M \backslash B(r)\right)>Q-1$ for any $r<R$. 
Let us denote by $\mathbf{M}$ the Brownian motion on $M$ and by $\mathbf{M}^{r}$ the absorbing Brownian motion on $M \backslash B(r)$. Let $\overline{\mathbf{M}^{r}}$ be the branching Brownian motion with motion component $\mathbf{M}^{r}$ and branching rate $\delta_{R}$ such that $Q(x) \equiv Q$. Theorem 3.4 and Remark 3.1 then imply the following: if $(Q-1) S(R) G(R)>\frac{1}{2}$ then $\overline{\mathbf{M}^{r}}$ extincts locally if and only if $r_{0} \leq r<R$, and if $(Q-1) S(R) G(R) \leq \frac{1}{2}$ then $\overline{\mathbf{M}^{r}}$ extincts locally for any $r<R$.

For instance, take the $d$-dimensional hyperbolic space as $M$ (see [7, Example 3.3] for a definition).

(i) For $d=2, S(R) G(R)$ is strictly increasing,

$$
\lim _{R \rightarrow 0} S(R) G(R)=0, \quad \text { and } \quad \lim _{R \rightarrow \infty} S(R) G(R)=1
$$

[15, Example 2.6]. Hence, if $Q>\frac{3}{2}$ then there exists a unique root, $R_{0}$, such that $(Q-1) S\left(R_{0}\right) G\left(R_{0}\right)=\frac{1}{2}$. Moreover, if $R>R_{0}$ then $\overline{\mathbf{M}^{r}}$ extincts locally if and only if $r_{0} \leq r<R$. If $R \leq R_{0}$ then $\overline{\mathbf{M}^{r}}$ extincts locally for any $r<R$. On the other hand, if $Q \leq \frac{3}{2}$ then $(Q-1) S(R) G(R)<\frac{1}{2}$ for all $R>0$ and $\overline{\mathbf{M}^{r}}$ extincts locally for any $r<R$.

(ii) For $d=3$,

$$
(Q-1) S(R) G(R)>\frac{1}{2} \quad \Longleftrightarrow \quad Q>2+\frac{1}{\mathrm{e}^{2 R}-1},
$$

by [15, Example 2.6]. Hence, if the right-hand side of (4.1) holds, then $\overline{\mathbf{M}^{r}}$ extincts locally if and only if $r_{0} \leq r<R$. Otherwise, $\overline{\mathbf{M}^{r}}$ extincts locally for any $r<R$.

(iii) For $d \geq 4, S(R) G(R)<1 /(d-1)$, by [15, Example 2.6]. Therefore, if $Q \leq(d+1) / 2$ then $\overline{\mathbf{M}^{r}}$ extincts locally for any $r<R$.

\subsection{Branching symmetric $\alpha$-stable processes}

Example 4.3. Suppose that $d=1$ and $1<\alpha \leq 2$. Let $D=\mathbb{R} \backslash\{0\}, \mu=\delta_{a}, a>0$, and $p_{0}(x)+p_{2}(x) \equiv 1$ on $D$. By combining Theorem 3.1 with [13, Example 3.3], we see that this branching symmetric $\alpha$-stable process extincts if and only if

$$
p_{2}(a) \leq \frac{1}{2}-\frac{\Gamma(\alpha) \cos (\pi \alpha / 2)}{4 a^{\alpha-1}} .
$$

Example 4.4. Suppose that $1<\alpha \leq 2$ and $d>\alpha$. Let $\delta_{r}$ be the surface measure of a sphere $\partial B(r)=\left\{x \in \mathbb{R}^{d}:|x|=r\right\}$. Take $\mu=\delta_{r}$ and assume that $Q(x) \equiv Q$. Using [18, Example 4.1], we see from Theorem 3.2 and Remark 3.1 that if $Q>1$ then $\overline{\mathbf{M}^{\alpha}}$ extincts locally if and only if

$$
0<r \leq\left\{\frac{\sqrt{\pi} \Gamma((d+\alpha) / 2-1) \Gamma(\alpha / 2)}{(Q-1) \Gamma((\alpha-1) / 2) \Gamma((d-\alpha) / 2)}\right\}^{1 /(\alpha-1)} .
$$

On the other hand, if $Q \leq 1$ then $\overline{\mathbf{M}^{\alpha}}$ extincts locally for any $r>0$.

Let $\overline{\delta_{r}}$ be the normalized surface measure of $\partial B(r)$, that is, $\overline{\delta_{r}}=\delta_{r} / \delta_{r}(\partial B(r))$. Take $\mu=\overline{\delta_{r}}$ and assume that $Q(x) \equiv Q$. Note that

$$
\lambda\left(\overline{\delta_{r}} ; \mathbb{R}^{d}\right)=\delta_{r}(\partial B(r)) \lambda\left(\delta_{r} ; \mathbb{R}^{d}\right)
$$

and that

$$
\delta_{r}(\partial B(r))=\frac{2 \pi^{d / 2} r^{d-1}}{\Gamma(d / 2)} .
$$


Thus, if $Q>1$ then $\overline{\mathbf{M}^{\alpha}}$ extincts locally if and only if

$$
r \geq\left\{\frac{(Q-1) \Gamma(d / 2) \Gamma((\alpha-1) / 2) \Gamma((d-\alpha) / 2)}{2 \pi^{(d+1) / 2} \Gamma(\alpha / 2) \Gamma((d+\alpha) / 2-1)}\right\}^{1 /(d-\alpha)} .
$$

On the other hand, if $Q \leq 1$ then $\overline{\mathbf{M}^{\alpha}}$ extincts locally for any $r>0$.

Example 4.5. Suppose that $0<\alpha \leq 2$ and $d>\alpha$. Let $\chi_{B(r)}(x) \mathrm{d} x$ be the $d$-dimensional Lebesgue measure restricted to the ball $B(r)=\left\{x \in \mathbb{R}^{d}:|x|<r\right\}$. Take

$$
\mu(\mathrm{d} x)=\chi_{B(r)}(x) \mathrm{d} x
$$

and assume that $Q(x) \equiv Q$. If $Q>1$ and

$$
0<r \leq\left\{\frac{2^{\alpha-1} \Gamma(d / 2) \Gamma(\alpha / 2+1)}{(Q-1) \Gamma((d-\alpha) / 2)}\right\}^{1 / \alpha},
$$

then $\overline{\mathbf{M}^{\alpha}}$ extincts locally, by [14, Example 3.10]. On the other hand, if $Q \leq 1$ then $\overline{\mathbf{M}^{\alpha}}$ extincts locally for any $r>0$.

\section{Acknowledgements}

The author would like to thank Professor Shinzo Watanabe for his comment which motivated the present work. He is grateful to Professor Zhen-Qing Chen for his suggestion which improved Example 4.4. His appreciation also goes to Dr Kaneharu Tsuchida for his advice. Finally, the author would like to express his sincere gratitude to Professor Masayoshi Takeda for his careful reading of this manuscript, invaluable advice, and suggestions.

\section{References}

[1] Albeverio, S. And MA, Z.-M. (1992). Additive functionals, nowhere Radon and Kato class smooth measures associated with Dirichlet forms. Osaka J. Math. 29, 247-265.

[2] Blumenthal, R. M. and Getoor, R. K. (1968). Markov Processes and Potential Theory. Academic Press, New York.

[3] Chen, Z.-Q. (2002). Gaugeability and conditional gaugeability. Trans. Amer. Math. Soc. 354, 4639-4679.

[4] Engländer, J. AND Kyprianou, A. E. (2004). Local extinction versus local exponential growth for spatial branching processes. Ann. Prob. 32, 78-99.

[5] Engländer, J. AND Pinsky, R. G. (1999). On the construction and support properties of measure-valued diffusions on $D \subseteq \mathbb{R}^{d}$ with spatially dependent branching. Ann. Prob. 27, 684-730.

[6] Fukushima, M., Oshima, Y. and Takeda, M. (1994). Dirichlet Forms and Symmetric Markov Processes (De Gruyter Stud. Math. 19). Walter de Gruyter, Berlin.

[7] GRIGOR'YAN, A. (1999). Analytic and geometric background of recurrence and non-explosion of the Brownian motion on Riemannian manifolds. Bull. Amer. Math. Soc. 36, 135-249.

[8] Ikeda, N., Nagasawa, M. and Watanabe, S. (1968). Branching Markov processes. I. J. Math. Kyoto Univ. 8, 233-278.

[9] Ikeda, N., Nagasawa, M. And Watanabe, S. (1968). Branching Markov processes. II. J. Math. Kyoto Univ. 8, 365-410.

[10] Pinsky, R. G. (1995). Positive Harmonic Functions and Diffusion: an Integrated Analytic and Probabilistic Approach. Cambridge University Press.

[11] Pinsky, R. G. (1996). Transience, recurrence and local extinction properties of the support for supercritical finite measure-valued diffusions. Ann. Prob. 24, 237-267.

[12] Sevast'yanov, B. A. (1958). Branching stochastic processes for particles diffusing in a bounded domain with absorbing boundaries. Theory Prob. Appl. 3, 111-126.

[13] Shiozawa, Y. (2004). Principal eigenvalues for time changed processes of one-dimensional $\alpha$-stable processes. Prob. Math. Statist. 24, 355-366. 
[14] Shiozawa, Y. and Takeda, M. (2005). Variational formula for Dirichlet forms and estimates of principal eigenvalues for symmetric $\alpha$-stable processes. Potential Anal. 23, 135-151.

[15] TAKedA, M. (2002). Conditional gaugeability and subcriticality of generalized Schrödinger operators. J. Funct. Anal. 191, 343-376.

[16] Takeda, M. (2006). Gaugeability for Feynman-Kac functionals with applications to symmetric $\alpha$-stable processes. Proc. Amer. Math. Soc. 134, 2729-2738.

[17] TAKeda, M. AND Tsuchida, K. (2006). Differentiability of spectral functions for symmetric $\alpha$-stable processes. To appear in Trans. Amer. Math. Soc.

[18] Takeda, M. And Uemura, T. (2004). Subcriticality and gaugeability for symmetric $\alpha$-stable processes. Forum Math. 16, 505-517.

[19] Watanabe, S. (1965). On the branching process for Brownian particles with an absorbing boundary. J. Math. Kyoto Univ. 4, 385-398. 